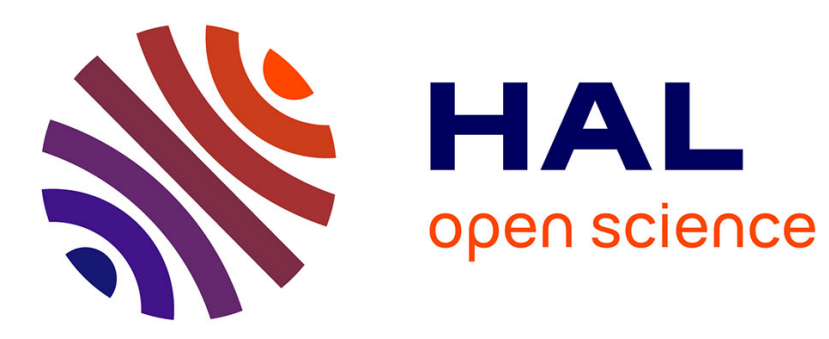

\title{
Anaphore et temps verbaux
}

\author{
Denis Apothéloz
}

\section{To cite this version:}

Denis Apothéloz. Anaphore et temps verbaux. Langue francaise, 2021, 210, pp.21-40. hal-03225650

\section{HAL Id: hal-03225650 \\ https://hal.science/hal-03225650}

Submitted on 7 Jun 2021

HAL is a multi-disciplinary open access archive for the deposit and dissemination of scientific research documents, whether they are published or not. The documents may come from teaching and research institutions in France or abroad, or from public or private research centers.
L'archive ouverte pluridisciplinaire HAL, est destinée au dépôt et à la diffusion de documents scientifiques de niveau recherche, publiés ou non, émanant des établissements d'enseignement et de recherche français ou étrangers, des laboratoires publics ou privés. 


\title{
Anaphore et temps verbaux
}

\author{
Denis Apothéloz \\ Université de Lorraine \& ATILF (CNRS UMR 7118)
}

\begin{abstract}
RÉSUMÉ
L'objectif de cet article est d'étudier les différentes facettes du fonctionnement anaphorique des temps verbaux. L'auteur distingue trois processus anaphoriques : (i) par ancrage temporel, (ii) par repérage et (iii) par relais. Il analyse le fonctionnement de quelques temps verbaux relativement à ces trois processus. Il montre que le repérage anaphorique ne concerne que certains temps, i.e. les temps composés ainsi que le conditionnel, tandis que l'ancrage temporel et le relais concernent tous les temps verbaux. Une attention particulière est accordée au plus-que-parfait dans les analepses, au conditionnel d'ultériorité ainsi qu'aux situations dans lesquelles un temps verbal proroge un contenu médiatif.
\end{abstract}

MOTS-CLÉS : temps verbal, plus-que-parfait, conditionnel, anaphore, anadeixis, médiativité

\section{ABSTRACT}

The aim of this article is to study the different aspects of the anaphoric functioning of tenses. The author distinguishes three anaphoric processes: via (i) temporal anchoring, (ii) localisation and (iii) relaying. He analyses the functioning of certain tenses in relation to the three processes, and shows that localisation concerns only certain of these, i.e. the compound tenses and the conditional, whereas temporal anchoring and relaying processes concern all tenses. Particular attention is paid to the pluperfect, especially in analepses, to the conditional of ulteriority, and situations in which a particular tense extends an evidential content.

KEYWORDS : tense, pluperfect, conditional, anaphora, anadeixis, evidentiality

\section{INTRODUCTION ${ }^{1}$}

Le but du présent article est de montrer l'intérêt que présentent les notions de référence et d'anaphore pour l'analyse des temps verbaux et, plus généralement, pour la représentation du temps dans le discours. Réciproquement, il s'agira également de mettre en évidence quel peut être l'apport des temps verbaux pour la compréhension des faits d'anaphore en général.

\section{2. temps verbaux, référence et anaphore}

On sait que le célèbre $\S 51$ de Reichenbach (1947) sur les temps verbaux prête à diverses interprétations concernant l'usage qui y est fait du terme de "référence ». Dans ce texte, en effet, le "point of reference » peut être interprété tantôt comme un repère, autrement dit comme un site temporel servant à localiser un autre site, tantôt comme un site auquel réfère (i.e. que désigne) la forme verbale. Dans un article commentant ce texte, Declerck (1986) montre de façon très convaincante que ce site peut être interprété tantôt comme un "time pointed from " (repère), tantôt comme un "time pointed to " (référence $)^{2}$. Or, la prise en compte de ces deux paramètres est un élément crucial dans la description des temps verbaux. Ce constat conduit à considérer que le modèle de Reichenbach est incomplet aussi longtemps qu'on ne lui adjoint pas au moins un paramètre supplémentaire - ce que je ferai ici (cf. Apothéloz 2017).

Je considérerai donc que la référence temporelle d'une forme verbale conjuguée est l'intervalle temporel que le grammème de temps verbal donne à voir du procès dénoté par le lexème verbal, « ce qui est perçu/montré du procès », pour reprendre l'expression

\footnotetext{
${ }^{1}$ Je remercie M. Nowakowska, W. De Mulder, F. Cornish ainsi que les relecteurs anonymes de leurs commentaires et conseils avisés sur des versions antérieures de cet article.

${ }^{2}$ Cette équivoque est favorisée par le fait que dans sa signification commune, le mot référence, en français comme en anglais, a les deux acceptions. L'équivoque porte d'autant plus à conséquence que les continuateurs et commentateurs de Reichenbach l'ont eux-mêmes perpétuée, souvent à leur dépens, certains ayant interprété le point $\mathrm{R}$ comme un repère (e.g. Comrie 1985), d'autres comme de la référence (e.g. McCawley 1981). La confusion perdure aujourd'hui encore.
} 
de Gosselin (2005 : 33). L'idée même que les temps verbaux réfèrent ne va pourtant pas de soi et a parfois été mise en doute. Ainsi, Kleiber (1993 : 157) considère que le rôle des temps verbaux ne consiste pas à identifier un intervalle temporel et que leur fonction référentielle relativement au procès n'est qu' "ancillaire ». Or, s'il est peu contestable que la localisation temporelle n'est qu'un élément du fonctionnement sémantique d'un verbe conjugué, il est non moins certain qu'elle est l'une des fonctions principales du grammème de temps verbal. À cet égard, une forme verbale conjuguée fonctionne à la manière d'une expression nominale, c'est-à-dire comme un nom déterminé par un grammème-article : l'élément lexical apporte sa dénotation, c'est-àdire, s'agissant d'un verbe, une signification sélectionnant un type de procès; et l'élément grammatical (le grammème du temps verbal) apporte les informations, temporelles et aspectuelles notamment, transformant le verbe en une forme apte à identifier et désigner une ou plusieurs instance(s) de ce procès et à lui (leur) assigner un site temporel. Que cette localisation soit des plus vagues et se limite à des indications d'époque («passé », «futur» ou «concomitant de l'énonciation») n'est pas un argument contre sa vertu référentielle, pas plus que son caractère ancillaire. Référer à un site temporel en le localisant dans une époque $e^{3}$ est certes considérablement plus abstrait et vague que référer à un chat sur un paillasson, mais ce n'en est pas moins référer, même si cette référence est au service de la désignation d'un procès.

Quant au caractère anaphorique des temps verbaux, du moins de certains d'entre eux, on oublie parfois qu'il a été mis en évidence dès les grammairiens de l'époque classique, avec la distinction entre temps «absolus» et temps « relatifs». Cette terminologie remonte à Girard (1747), mais la distinction se trouve déjà dans la Grammaire de Port-Royal (Arnauld \& Lancelot 1660). Le texte de Girard mérite d'être rappelé, tant il est clair à cet égard :

Lorsqu'ils [les temps verbaux] représentent le temps de l'événement par la seule comparaison avec celui où l'on parle, ils sont TEMPS ABSOLUS: lorsqu'ils le représentent par une double comparaison, faite non seulement avec le temps de la parole mais encore avec celui de quelqu'autre événement, ils sont TEMPS RELATIFS. (Girard, 1747, tome 2, p. 25)

La «double comparaison» serait aujourd'hui formulée en utilisant la notion de repérage : repérage par rapport au moment de l'énonciation, d'une part, et repérage par rapport à un « autre événement », d'autre part - l'énonciation et l'autre événement ayant la fonction de repère. Une autre terminologie consiste à dire que le premier repérage est déictique, le second anaphorique ${ }^{4}$.

Il apparaît donc quelque peu excessif d'attribuer à McCawley (1971) ou à Partee (1973, 1984), comme on le fait parfois, la paternité de l'utilisation de la notion d'anaphore dans la description des temps verbaux. Certes Girard n'utilise pas le terme d' " anaphore », mais la notion est bel et bien présente dans son texte, et de façon on ne peut plus claire. L'originalité de McCawley et de Partee réside surtout dans les analyses du parallélisme entre pronoms et temps verbaux.

Si le caractère anaphorique de certains temps verbaux, comme l'imparfait ou le passé simple, a fait l'objet de diverses polémiques, il n'en va pas de même pour d'autres temps, comme le plus-que-parfait ou le conditionnel. Ce sont ces temps verbaux qui vont d'abord m'intéresser ici. Dans la continuité de Girard, Comrie (1985) les décrit comme des temps «absolus-relatifs », i.e. combinant repérage déictique et repérage anaphorique.

\footnotetext{
${ }^{3}$ J'emprunte à Koschmieder (1996) l'expression de « site temporel », qui traduit l'allemand Zeitstellenwert.

${ }^{4}$ Beauzée (1765) parle quant à lui d' « époque de comparaison». Les qualificatifs d' « absolu» et de « relatif» ont donné lieu à diverses critiques, de même que la possibilité même de distinguer entre temps absolu et temps relatif. Sur les usages de cette terminologie, voir par ex. Yvon (1951) et Vetters (1996).
} 


\section{ANTÉRIORITÉ ET ULTÉRIORITÉ}

\subsection{Le plus-que-parfait et l'analepse}

Comme tous les temps composés du français ${ }^{5}$, le plus-que-parfait est susceptible, selon le contexte, d'orienter la référence temporelle vers deux phases distinctes du procès signifié par le verbe : la phase processive (i.e. le procès proprement dit) et la phase résultative, ou état résultant. Les grammaires traditionnelles appellent respectivement « inaccompli» et «accompli» ces deux interprétations, qui sont illustrées par les formes en gras des extraits (1) et (2):

1) Puis, on était passé dans la pièce d'à côté : on s'était assis autour d'une table, on avait mangé des gâteaux onctueux, des mousses, des salades de fruits, bu du champagne. $(\mathrm{H}$. Bianciotti, Sans la miséricorde du Christ, 1985)

2) Dix-huit mois venaient de suffire : le ménage des Roubaud s'était gâté, le mari avait mangé les cinq mille francs au jeu, la femme en était arrivée à prendre un amant, pour se distraire. (E. Zola, La Bête humaine, 1890)

Les plus-que-parfaits de (1) sont processifs : ils visent à désigner, de la même manière que des passés simples, une séquence de procès se succédant les uns aux autres. Cette interprétation est favorisée par l'adverbe de consécution (puis) par lequel débute l'extrait. Cependant, avec le plus-que-parfait, et contrairement à ce que produirait un passé simple, cette séquence s'inscrit dans une «antériorité dans le passé »: elle présuppose que d'autres procès ont été précédemment évoqués (ici hors-champ par l'artifice du découpage de l'extrait), eux aussi passés mais postérieurs à ceux désignés dans (1). Les temps verbaux sont ici clairement anaphoriques et forment une analepse : ils concernent une séquence narrative secondaire ${ }^{6}$, se déroulant antérieurement à la narration principale.

Il en va différemment dans (2). La séquence qui suit les deux-points vise à désigner non pas des procès successifs, mais différentes facettes de l'état dans lequel se trouve, dix-huit mois après certains événements, le ménage des Roubaud, état consécutif à certains procès. Chacun de ces plus-que-parfaits concerne une facette de cet état : le ménage en général (qui est gâté), les économies (qui sont mangées) et l'épouse (qui $a$ un amant). Ces plus-que-parfaits sont tous résultatifs, et il n'y a entre les états qu'ils décrivent aucune relation de succession temporelle : ils sont synchrones.

Seule l'interprétation processive du plus-que-parfait, comme dans (1), nous retiendra ici. En utilisant une version amendée du modèle de Reichenbach (1947), distinguant repère et référence, on peut représenter comme suit les propriétés aspectuo-temporelles du grammème de plus-que-parfait dans son interprétation processive :

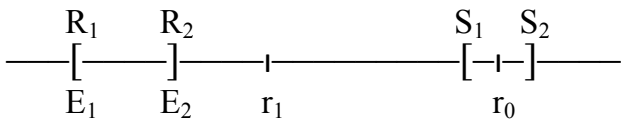

Figure 1 : Chronogramme du plus-que-parfait processif

$\left[S_{1}-S_{2}\right]$ représente l'intervalle $S$ de l'énonciation, $\left[E_{1}-E_{2}\right]$ l'intervalle $E$ du procès dénoté par le verbe, et $\left[R_{1}-R_{2}\right]$ l'intervalle $R$ de la référence temporelle. $R$ est ici temporellement coextensif de $\mathrm{E}$, ce qui correspond à l'interprétation processive du temps verbal. (En interprétation résultative, R serait postérieur à E.) Dans $\mathrm{S}$ se trouve un

\footnotetext{
5 À l'exception du passé antérieur, que les aspectologues s'accordent à considérer comme un temps toujours résultatif.

${ }^{6}$ Qualificatif à comprendre ici seulement au sens d'« enchâssée ».
} 
premier repère : $r_{0}$. C'est relativement à ce repère que le second repère, $r_{1}$, est localisé dans le passé. L'inclusion de $\mathrm{r}_{0}$ dans $\mathrm{S}$ rend compte de la dimension déictique du plusque-parfait ; $r_{0}$ correspond donc à ce que Wilmet (1997) appelle " repère d'actualité » et que cet auteur distingue du moment de l'énonciation ${ }^{7}$. Quant au repère $r_{1}$, il permet de localiser $\mathrm{R}$, et par conséquent $\mathrm{E}$, comme antérieurs à lui-même. Il rend donc compte de la dimension anaphorique du plus-que parfait.

Dans l'extrait (1) tel qu'il a été découpé, aucune expression ne permet d'ancrer temporellement $r_{1}$. Mais dans le texte original cette expression existe : c'est le dernier passé simple de la narration principale. On reviendra plus loin sur cette notion d'ancrage, qui relève elle aussi du mécanisme de l'anaphore.

Le repère $r_{1}$ comporte donc deux informations temporelles : d'une part, sa propre localisation, déterminée déictiquement comme antérieure à $\mathrm{r}_{0}$ (donc passée); d'autre part, la localisation de la référence du temps verbal (R), située anaphoriquement comme antérieure à $\mathrm{r}_{1}$. La mesure de cette antériorité peut être indiquée par des expressions anaphoriques comme la veille, trois jours plus tôt, etc. Ce repère est donc constitutif de la « carte sémantique » du plus-que-parfait, de sorte que l'utilisation de ce temps verbal est pragmatiquement inappropriée si le contexte où il est produit n'est pas apte à lui fournir un ancrage temporel (Bertinetto 1986).

Cette analyse montre que le grammème de plus-que-parfait est anadéictique. Toutefois la cohabitation entre anaphore et deixis diffère ici de ce que décrit Ehlich (1982), pour qui il existe un continuum deixis-anaphore, et donc des expressions associant, à des degrés variables, déicticité et anaphoricité. Le plus-que-parfait comporte un premier composant exclusivement et pleinement déictique (le repérage de $\mathrm{r}_{1}$ à partir de $\mathrm{r}_{0}$ ), et un second composant exclusivement et pleinement anaphorique (le repérage de $\mathrm{R}$ à partir de $\mathrm{r}_{1}$ ). De surcroît, ces deux repérages sont ordonnés, le repérage déictique précédant le repérage anaphorique.

Arrêtons-nous sur le phénomène de l'analepse. Dans une séquence comme (1), le plus-que-parfait marque de façon continue que les événements narrés sont antérieurs à $r_{1}$. Il exprime et à la fois proroge cette information, par conservation de ce repère, qui demeure constant de verbe en verbe aussi longtemps que ce temps verbal est maintenu. Ce mécanisme relève de l'anaphore. La conservation de $r_{1}$ assure la «mémoire » du dispositif analeptique.

Or, il a été observé que lorsque les analepses sont d'une certaine longueur, il arrive que le plus-que-parfait processif soit inopinément abandonné pour un autre temps narratif, généralement le passé simple ou le passé composé (Barceló \& Bres 2006) ${ }^{8}$. Voici une illustration de ce tense-switching ${ }^{9}$. Les formes analeptiques sont en gras, les passés simples analeptiques en italiques grasses :

3) Il me dit que les choses avaient mal tourné là-bas [...]. Sur le coup de sept heures, deux petits gars qu'on avait chargés de surveiller le dépôt d'armes avaient aperçu trois cyclistes allemands en casques et mitraillettes en bandoulière [...]. Et les petits gars [...] avaient lâché deux rafales de mitrailleuse devant eux, au hasard ; aussitôt les Allemands sautèrent à terre en abandonnant leurs bicyclettes, s'aplatirent dans le fossé et commencèrent à faire feu.

[suivent 30 lignes de passés simples]

Puis ils [les deux petits gars] repartirent, regagnèrent la route et, silencieux, harassés,

\footnotetext{
${ }^{7}$ On distingue donc ici, d'une part l'énonciation comme événement situé dans l'espace et le temps, d'autre part son utilisation comme repère. Par défaut - mais par défaut seulement - l'énonciation est utilisée comme repère $\left(\mathrm{r}_{0}\right.$ est situé dans S). Cette distinction permet de rendre compte de phénomènes bien connus de transposition temporelle, comme le présent narratif ( $\mathrm{r}_{0}$ est projeté dans le passé) ou le passé épistolaire ( $\mathrm{r}_{0}$ est projeté dans le futur) en restant sur un terrain purement aspectuo-temporel et en faisant l'économie d'explications psychologisantes.

${ }^{8}$ Quelques études ont été consacrées à ce phénomène (cf. Combettes 2008, Apothéloz 2018), qui a également été observé en espagnol (Lhafi 2016).

${ }^{9}$ Terme parfois utilisé en linguistique anglaise pour décrire des faits variationnels analogues, par exemple concernant le simple past et le present perfect (e.g. Portero Muñoz 2018).
} 
reprirent en sens inverse [...]. Naturellement, les Allemands avaient mis la main sur le dépôt d'armes et, quelques instants après, ils étaient des centaines à explorer, mitraillette sous le bras, les abords des fourrés où ils avaient trouvé les containers et les parachutes soigneusement roulés et ficelés. [...]

- Les foutus imbéciles! s'écria Max quand je l'eus mis au courant de la situation. (L.-R. des Forêts, Un malade en forêt, 1985)

L'analepse débute ici à la faveur d'un discours rapporté, et elle est formée de l'intégralité des propos rapportés. À partir de l'adverbial sur le coup de sept heures, ce rapport est formulé sur le mode indirect libre. On observe cependant que le plus-queparfait est assez vite abandonné au profit du passé simple (cf. les Allemands sautèrent à terre...), alors même qu'on se trouve à l'intérieur de l'analepse et donc dans une narration secondaire. Ce n'est qu'après une longue séquence de passés simples que le texte revient au plus-que-parfait.

Ce changement de temps verbal peut avoir toutes sortes de motivations, certaines liées aux propriétés aspectuo-temporelles du plus-que-parfait, d'autres à la conduite même du cours narratif - questions qu'il n'est pas possible d'aborder ici. Quoi qu'il en soit, du point de vue de la gestion des anaphores, cet abandon du plus-que-parfait produit un « oubli » d'une information importante : l'antériorité. La mémorisation de la temporalité analeptique (i.e. l'anaphore associée à $\mathrm{r}_{1}$ ) n'est plus marquée grammaticalement; elle est laissée à la mémoire du lecteur, ce dernier pouvant d'ailleurs recourir à d'autres indices pour s'orienter temporellement dans le texte.

\subsection{Le conditionnel et la prolepse}

Quand il est temporel, i.e. sans signification modale (hypothétique, contrefactuelle, atténuative, etc.), le conditionnel exprime l'ultériorité du passé. Son fonctionnement présente alors une certaine symétrie avec celui du plus-que-parfait processif. On lui reconnaît deux contextes d'emplois ${ }^{10}$.

Dans le premier, il est « subjectif», pour reprendre le qualificatif utilisé par NilssonEhle (1943). Il exprime le point de vue d'une instance (un sujet de conscience) autre que l'énonciateur, comme c'est typiquement le cas dans le discours indirect (lié ou libre) ou dans toute autre formulation où l'ultériorité est associée à la médiation d'un point de vue. Illustration :

4) Ils parlaient de ce qu'ils feraient plus tard, quand ils seraient sortis du collège. D'abord, ils entreprendraient un grand voyage avec l'argent que Frédéric prélèverait sur sa fortune, à sa majorité. Puis ils reviendraient à Paris, ils travailleraient ensemble, ne se quitteraient pas [...]. (G. Flaubert, L'Éducation sentimentale, 1869)

On qualifiera de «médiatifs » les contextes qui déclenchent cette interprétation ${ }^{11}$, et l'on désignera par le terme de « médiateur » (Desclés \& Guentchéva 2000) l'instance le sujet de conscience - dont la subjectivité est représentée : dans (4), Frédéric et son ami, désignés par ils.

Dans le second contexte, il est " objectif» en ceci que les énoncés où il figure sont entièrement pris en charge par l'énonciateur. L'effet produit est une prolepse (anticipation) au sens de Genette (1972). Cet emploi est réputé fréquent dans les biographies et les textes des historiens. En voici un exemple :

5) Juju était le chef de la «base ouvrière» de Sochaux. Un petit râblé, tout en muscles compacts et voix rauque, un petit matelot qui aurait bien plu à Genet. Quelques années plus tard, il se tuerait magnifiquement sur une route de cette contrée que la rigueur de ses

\footnotetext{
${ }^{10}$ Le futur et les formes prospectives (il allait pleuvoir, il devait pleuvoir) permettent, avec diverses nuances, de produire la même expression de l'ultériorité.

1 Dans la tradition logique, on qualifie ces contextes d' «intensionnels » (avec un 's') (Russell 1905) ou d'« opaques » (Quine 1960).
} 
hivers fait nommer "petite Sibérie » : sa voiture faucherait tout un bosquet de sapins avant de s'arrêter, embrochée par le toit sur un tronc cassé. (O. Rolin, Tigre en papier, 2002)

Plusieurs auteurs (e.g. Korzen \& Nølke 2001, Azzopardi 2011) ont noté que ces deux variantes de l'ultériorité ont des propriétés différentes. La principale différence concerne la localisation temporelle du procès, donc la référence temporelle du temps verbal.

Avec la variante subjective, la référence temporelle n'est soumise à aucune contrainte d'époque: elle peut être passée, présente ou future. Avec la variante objective, en revanche, elle ne peut être localisée que dans le passé. Cependant, dans les deux cas elle est située ultérieurement à un repère $\left(r_{1}\right)$ localisé dans le passé. Dans (4), ce repère est ancré sur la référence temporelle fournie par le verbe introducteur de discours rapporté; dans (5), il trouve son ancrage dans l'antécédent temporel de l'expression quelques années plus tard.

Au plan aspectuel, le conditionnel comme le futur neutralise la distinction entre visée perfective et imperfective (Gosselin 1996), et le choix de l'une ou l'autre de ces interprétations dépend de divers facteurs contextuels (le lexème verbal étant le premier de ces facteurs). Pour simplifier et parce que la distinction est peu pertinente ici, on figurera sous forme de chronogramme seulement l'interprétation perfective :

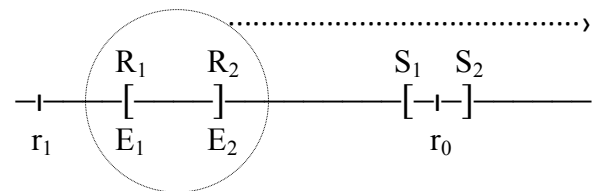

\section{Figure 2 : Chronogramme du conditionnel d'ultériorité}

Le cercle et la flèche en pointillés indiquent l'empan de localisation du procès et ne concernent donc que la variante subjective. Le caractère déictique du conditionnel d'ultériorité réside dans l'information « passé », donc dans l'antériorité de $\mathrm{r}_{1}$ par rapport à $\mathrm{r}_{0}$; le caractère anaphorique, dans le fait que la référence du temps verbal $(\mathrm{R})$ est ultérieure à $r_{1}$. De façon symétrique avec le plus-que-parfait, la mesure de cette ultériorité peut être précisée (par des anaphoriques comme le lendemain, trois jours plus tard, etc.). Ainsi, le conditionnel d'ultériorité apparaît lui aussi comme un temps verbal anadéictique, et les deux composants déictique et anaphorique y cohabitent de la même manière que dans le cas du plus-que-parfait, et avec la même priorité de la deixis sur l'anaphore.

Cependant, si les deux variantes subjective et objective du conditionnel d'ultériorité sont des anaphoriques temporels, la variante subjective est anaphorique pour une autre raison encore : non seulement elle conserve l'information temporelle associée à $r_{1}$, mais elle relaie la subjectivité propre au contexte médiatif. Dans (4), la persistance du conditionnel fonctionne comme un marqueur prorogeant ces deux types d'informations. À ce titre, elle inclut également les médiateurs désignés par ils.

Vue sous cet angle, l'anaphoricité du conditionnel subjectif apparaît comme particulièrement complexe, cumulant anaphore temporelle et anaphore médiative. Rétrospectivement, on peut faire le même constat pour le plus-que-parfait quand il se trouve en contexte médiatif, ce qui est le cas dans l'exemple (3) supra. 
Parfois l'ancrage temporel de $\mathrm{r}_{1}$ n'est donné que très indirectement et doit être récupéré par inférence, d'une façon qui s'apparente à la recherche d'un antécédent ${ }^{12}$ en cas d'anaphore associative :

6) Du père je ne voyais que le dos énorme. Courbé sur la serrure, il fermait la porte à clef. La maison resterait vide et noire jusqu'à leur retour. (J.-P. Sartre, La Nausée, 1938)

L'ultériorité de la référence temporelle de la maison resterait vide et noire doit être calculée par rattachement à la référence temporelle de il fermait la porte à clef, expression donnant son ancrage, son interprétant temporel au repère $r_{1}$ du conditionnel. On notera au passage que cet exemple est susceptible d'une lecture aussi bien objective que subjective. Interprété objectivement, le dernier énoncé est une anticipation entièrement prise en charge par l'énonciateur; interprété subjectivement, cette anticipation est une pensée attribuée au père en train de fermer la porte.

Selon Bres (2012), la variante objective du conditionnel d'ultériorité est diachroniquement récente; on ne la rencontre guère avant le début du $\mathrm{XIX}^{\mathrm{e}}$ siècle. Elle est généralement sporadique et concerne une séquence brève d'un ou deux verbes, comme dans (5)-(6). Quelques travaux ont toutefois observé une tendance récente, chez certains auteurs, à cumuler ce type de conditionnel, parfois en alternance avec un autre temps narratif (Wilhelm 2009, Ašić et al. 2017). Cette tendance a par exemple été observée dans les romans de J. Echenoz ou de M. Houellebecq.

\section{L'IMPARFAIT ET LA MÉDIATIVITÉ}

De façon générale, l'emploi d'un temps verbal peut être caractérisé de "médiatif» quand il relaie, ou contribue à relayer, un sujet de conscience distinct de l'énonciateur. Les manifestations les plus caractéristiques de ce fonctionnement sont les représentations de paroles, pensées ou perceptions qui ne sont pas celles de l'énonciateur actuel.

Les affinités de l'imparfait avec la médiativité sont connues depuis longtemps. Bally (1912) lui-même qualifiait de "subjectifs » les emplois de l'imparfait en contexte de discours indirect libre. Cependant, lorsque ce phénomène se produit, le temps verbal n'est généralement qu'un indice parmi d'autres. La signification même du verbe ainsi que d'autres éléments participent presque toujours à la construction de cette interprétation. Cela est tout à fait clair dans l'exemple suivant, où l'expression en italiques grasses prépare le lecteur à recevoir comme médiative la description qui la suit.

7) Bientôt, tous les sièges furent occupés. Elle jetait des regards autour d'elle en évitant qu'ils se posent sur le policier en civil. Visages harassés de ceux qui attendent leur train. Une femme répandait une odeur de poudre qui se mêlait à celle du tabac froid. Sur le mur du fond une affiche aux couleurs blanche et bleu ciel : un skieur glissait seul, au milieu d'une grande étendue de neige qui réverbérait le soleil. Et il était écrit : VACANCES EN ENGADINE. (P. Modiano, Une jeunesse, 1981)

Dans ce texte, l'effet de perception représentée est accru - pour ne pas dire confirmé - par le fait que le contenu narratif d'une affiche (une représentation visuelle) est décrit avec le même statut de réalité que l'environnement concret de la médiatrice (elle). La

\footnotetext{
${ }^{12}$ Je reprends ici par commodité ce terme d'« antécédent », qui a donné lieu à diverses critiques. Tesnière (1969) par exemple lui préférait celui de "source sémantique », qui présente notamment l'avantage de mettre sous une même dénomination anaphore et cataphore. Cornish (1987 et ici même) parle quant à lui d'«antecedent-trigger» (déclencheur d'antécédent).
} 
prorogation de cet effet interprétatif peut être considérée comme relevant de l'anaphore, et l'imparfait, comme plus haut le conditionnel, en est l'un des marqueurs ${ }^{13}$.

On caractérise communément l'anaphore comme un fait de dépendance interprétative relativement à une information préalablement donnée dans le même discours (Halliday \& Hasan 1976) ${ }^{14}$. Or, s'agissant des anaphores médiatives, cette dépendance est assez particulière.

D'une part, elle ne se manifeste pas, comme c'est typiquement le cas des anaphores, par une incomplétude de la signification de l'anaphorique. C'est la raison pour laquelle le phénomène peut passer inaperçu - et, de fait, passe souvent inaperçu. Quand cette situation se produit, une signification présentée "subjectivement» est reçue « objectivement », souvent sans qu'il y ait réelle atteinte à l'intelligibilité du texte.

Mais d'autre part, il peut arriver que la non-saisie de la médiativité fasse apparaître certains énoncés comme empiriquement aberrants ou paradoxaux. C'est alors l'aberration ou le paradoxe qui incite à "subjectiviser » l'expression, comme c'est partiellement le cas dans (7) avec la description de l'affiche. On y reviendra brièvement plus loin (§ 5.4.).

Les temps verbaux peuvent cependant manifester des formes plus discrètes de médiativité. Un emploi impliquant encore une fois l'imparfait, à ma connaissance très peu étudié, est celui qu'on rencontre dans certains résumés ou comptes rendus de narrations, et qu'on appellera ici « méta-narratif» (Apothéloz, 2021). Baranzini \& Ricci (2015) ont décrit ce phénomène à propos de l'imperfetto italien (voir aussi Baranzini 2019), mais l'imparfait français partage le même emploi. L'une des raisons pour lesquelles il a si peu attiré l'attention est probablement sa ressemblance avec l'imparfait narratif, auquel il est parfois identifié. En voici un exemple :

8) [Article sur le roman de V. Despentes, Vernon Subutex 3, paru en 2017] La description de la brutalité générale de l'époque [...] est cependant, depuis le départ, au cœur de Vernon Subutex. Résumé des épisodes précédents : dans le tome 1, l'ex-disquaire Vernon Subutex se retrouvait à la rue après la mort d'un ami qui payait son loyer. Tandis qu'il passait du canapé d'une connaissance à l'autre, on traversait Paris, toutes les couches de la société, tout le spectre sociologique et politique, et l'on découvrait des personnages perclus de solitude. Vernon finissait SDF. Dans l'épisode 2, d'une étrange douceur, les anciens hébergeurs de Vernon se mettaient à former un vague groupe autour de lui. Ils se réunissaient au parc des Buttes-Chaumont au côté de cet extraordinaire DJ qui les faisait danser.

Quand s'ouvre le tome 3, les mois ont passé [...]. (Le Monde, 26.05.2017)

Que désignent au juste ces énoncés à l'imparfait? Certainement pas les procès que dénotent les verbes concernés - du moins pas directement. En fait, la généralisation de l'imparfait dans ce résumé produit une narration de narration, une narration « seconde ». La référence de toute la séquence est constituée non pas des événements et situations désignés, mais de leur désignation antérieure dans une narration "première ». Et le fait d'utiliser un temps du passé produit, comme effet associé, que cette narration première est en réalité la réception antérieure (ou une réception antérieure) des tomes 1 et 2 du roman dont il est question. C'est en quelque sorte la mémoire de cette réception qui est suggérée par ces imparfaits, ce que confirment d'ailleurs certaines expressions, comme on traversait Paris [...] et l'on découvrait des personnages perclus de solitude, où le pronom peut être interprété comme désignant un lecteur quelconque.

On est donc très clairement ici dans un contexte médiatif, mais avec un type tout à fait particulier de médiativité, dans lequel le médiateur n'est pas un individu clairement

\footnotetext{
${ }_{13}^{13}$ Sur la question des temps verbaux et de la médiativité, voir Guentchéva (1994).

14 On définit souvent aussi l'anaphore comme une relation de présupposition. Voir cette définition de M.-J. Béguelin : «[...] les expressions dites anaphoriques ont pour propriété commune de désigner un référent en le présupposant déjà validé dans la mémoire discursive [...] » (Béguelin $2000: 291$, les italiques sont de l'auteure).
} 
identifiable. S'agit-il de cette abstraction qu'on appelle " instance narratrice »? ou d'un lecteur générique? ou du lecteur qu'a nécessairement été antérieurement l'énonciateur actuel du résumé ? Quoi qu'il en soit, le maintien de l'imparfait contribue dans cet extrait à relayer cette lecture médiative, et comporte à ce titre une dimension anaphorique ${ }^{15}$.

\section{L'ANAPHORE ET LES TEMPS VERBAUX}

Considérer l'anaphore sous l'angle de la dépendance interprétative rend compte du point de vue de la réception. Pour intégrer le point de vue de la production, on considérera complémentairement l'anaphore comme un mécanisme de prorogation : un anaphorique dépend interprétativement d'une information apportée antérieurement et, parce qu'il intègre cette information, la proroge dans le discours.

Cette définition conduit à distinguer, sur la base des faits présentés aux $\S 3-4$, trois processus anaphoriques.

\subsection{Processus d'ancrage temporel}

Il a plusieurs fois été question d'ancrage dans les analyses ci-dessus. Ce processus concerne plusieurs éléments dans le fonctionnement des temps verbaux.

Il a tout d'abord été évoqué à propos du repère $r_{1} d u$ plus-que-parfait et $d u$ conditionnel temporel. Ces deux temps imposent à ce repère d'être localisé déictiquement dans le passé, i.e. antérieurement à $\mathrm{r}_{0}$. Mais en discours, le site temporel de ce repère doit être spécifié contextuellement. C'est ce processus de spécification que désigne le terme d'ancrage. Par exemple, dans (3) et (4) supra, c'est le verbe introducteur de discours rapporté qui ancre temporellement $r_{1}$, site temporel par rapport auquel la référence des plus-que-parfaits processifs est antérieure (ex. 3), et celle des conditionnels, ultérieure (ex. 4). Cette spécification peut être un fait de pure interprétation, sans support d'aucune expression.

Cependant, la référence temporelle de ces temps verbaux, i.e. la localisation de $\mathrm{R}$, respectivement antérieur et ultérieur à $\mathrm{r}_{1}$, requiert elle aussi un ancrage, une spécification contextuelle. Dans (4), cette spécification est explicitement apportée par l'expression plus tard, quand ils seraient sortis du collège. Pour le premier plus-queparfait de (3), elle requiert la connaissance du texte antérieur; pour les suivants elle obéit à la logique interne du cours narratif et à la progression de $\mathrm{R}$ que ce cours implique, en fonction des procès évoqués. Plus-que-parfait et conditionnel temporel requièrent donc un double ancrage.

Les temps verbaux qui ne comportent pas de repère $r_{1}$ requièrent un seul ancrage, celui de leur référence temporelle. Grammaticalement, ils ne donnent comme information de localisation qu'une indication d'époque, en elle-même insuffisante ou sous-déterminée, comme cela a été noté par plusieurs auteurs (e.g. Bres 2007, qui parle de localisation «floue»; Gosselin 2017, 2021, pour qui l'intervalle de référence est intrinsèquement anaphorique car non autonome) ${ }^{16}$. En discours, cette information est spécifiée contextuellement. C'est ce qui se passe pour les passés simples de (3), par exemple, dont l'interprétation référentielle est soumise à la logique interne du cours narratif. C'est précisément ce processus que McCawley (1971) et Partee (1973) avaient comparé au fonctionnement des pronoms - cf. l'exemple Sheila had a party last Friday and Sam got drunk (Partee 1973: 605), où le second verbe hérite de la référence temporelle du premier, elle-même localisée par un adverbial. Les débats sur la question

\footnotetext{
${ }^{15}$ Les analyses de L. de Saussure (2003 : 240), selon lesquelles l'imparfait narratif consiste en un usage interprétatif et non pas descriptif du temps verbal (au sens de Sperber \& Wilson 1989:343), suggèrent que la différence entre l'imparfait narratif et cette variante médiative est peut-être plus ténue qu'il n'y paraît.

${ }^{16}$ Cette généralité ne concerne pas le participe présent (cf. infra).
} 
de savoir si les temps verbaux, du moins certains d'entre eux, sont ou ne sont pas anaphoriques concernent en général ce processus d'ancrage ${ }^{17}$.

L'ancrage se fait donc au moyen d'expressions susceptibles de fonctionner comme des antécédents temporels, permettant de calculer le site temporel visé. La coréférence temporelle n'est alors qu'un cas particulier: en contexte narratif, par exemple, la référence temporelle tend à progresser aussi longtemps que le temps verbal est perfectif, de sorte que l'anaphore est indissociablement temporelle et associative.

Il est intéressant de noter que, dès ce niveau, deixis et anaphore sont associées comme deux processus qui se succèdent.

On pourrait introduire ici une distinction supplémentaire. C'est celle entre les temps verbaux qui comportent une information d'époque, comme le futur ou l'imparfait, et ceux qui n'en comportent pas. Ainsi, un participe présent ne donne aucune information d'époque. Mais, dès lors qu'il est utilisé, il est nécessairement ancré temporellement. Le participe présent est donc exclusivement anaphorique, et en principe toujours soumis à ce processus d'ancrage.

\subsection{Processus de repérage anaphorique}

Le deuxième processus ne concerne pas tous les temps verbaux. Il s'agit de celui qui est associé au repère $r_{1}$ tel qu'il fonctionne avec le plus-que-parfait processif et le conditionnel d'ultériorité (pour ne mentionner que ces deux temps). Il concerne donc les temps « relatifs » au sens de Comrie (1985).

Rappelons que deux informations temporelles sont associées au repère $r_{1}$ :

- d'une part, sa propre localisation qui, comme nous l'avons vu, est d'abord déterminée déictiquement, puis spécifiée anaphoriquement par ancrage ;

- d'autre part, la localisation de R.

$\mathrm{r}_{1}$ exprime une relation temporelle entre ces deux sites : celle d'antériorité (plus-queparfait) ou d'ultériorité (conditionnel).

C'est ce second processus qui est appelé ici « repérage anaphorique $»^{18}$. L'intervalle impliqué par cette relation est souvent spécifié au moyen d'adverbiaux, comme la veille, quelques semaines auparavant (pour le plus-que-parfait) ou le lendemain, quelques semaines plus tard (pour le conditionnel), expressions qui «quantifient» l'antériorité ou l'ultériorité. Il est rare qu'un conditionnel d'ultériorité objectif soit utilisé sans ce type d'adverbial.

On voit la différence entre ces deux premiers processus. Tandis que l'ancrage consiste dans la spécification contextuelle d'une information temporelle au départ sousspécifiée, le repérage anaphorique consiste en une information intrinsèquement anaphorique parce que purement relationnelle. Tandis que l'ancrage est un processus interprétatif de contextualisation, fondamentalement contingent et mobilisant au décodage la recherche d'antécédents temporels, le repérage anaphorique n'est ni interprétatif ni contingent : il est «systémique », il est un élément de ce que code le grammème de temps verbal.

La distinction de ces deux processus montre que les temps verbaux ne sont pas tous anaphoriques de la même manière, ni surtout avec la même complexité. Certains ne le sont que par ancrage temporel : tel est le cas du passé composé aoristique, du passé

\footnotetext{
${ }^{17}$ Pour une description des principes guidant ce processus, voir Gosselin (2005: 142 et suiv.). Une différence importante entre pronoms et temps verbaux, à ma connaissance rarement signalée, est que les grammèmes des temps verbaux ne sauraient être considérés comme des pro-formes. L'anaphoricité des temps verbaux a donné lieu à une multitude d'articles : e.g. Tasmowski-De-Ryck (1985), Hinrichs (1986), Berthonneau \& Kleiber (1993), Kleiber (1993, 2003), Molendijk (1996), Keromnes (1998), Vet (1996a,b, 1999), Vetters (1993, 1998), De Mulder \& Vetters (1999), Gosselin (2005), Bres (2007). Le $\mathrm{n}^{0} 1$ des Cahiers Chronos (1996) est principalement consacré à la question de l'anaphore et des temps verbaux.

${ }^{18}$ S'agissant d'un processus anaphorique, cette appellation peut paraître redondante. Elle est cependant nécessaire, car un site temporel peut être repéré déictiquement.
} 
simple ou du futur. C'est alors seulement la référence temporelle qui est soumise à ce processus. D'autres temps verbaux sont anaphoriques à un double titre : par ancrage temporel et par repérage anaphorique. Tel est le cas des temps qui, comme le plus-queparfait ou le conditionnel d'ultériorité, impliquent un repère $r_{1}$.

\subsection{Processus de relais}

Les deux processus décrits ci-dessus concernent une information de localisation temporelle. Il en va différemment du troisième processus. Il s'agit de la capacité qu'ont les temps verbaux, du moins certains d'entre eux, à hériter localement d'une interprétation médiative et à la relayer dans le discours. Nous avons observé ce phénomène avec le plus-que-parfait, le conditionnel et l'imparfait. Ainsi, dans l'extrait (4) supra, la répétition du conditionnel, après une première occurrence en contexte explicite de discours rapporté (ils parlaient de ce qu'ils feraient plus tard...), a pour effet de relayer cette information en la reportant sur les énoncés qui suivent. Et dans (7), la répétition de l'imparfait, suite à un énoncé indiquant une perception visuelle (elle jetait des regards autour d'elle), produit un effet analogue. Le type des procès désignés, de même que d'autres indices, contribuent certes à conforter cette interprétation, mais la réitération du même temps verbal n'en est pas moins un marqueur décisif. Dans la mesure où il est prorogatif et mémoriel, ce processus relève de l'anaphore. Il diffère des précédents sur deux points : (i) l'information qu'il proroge n'est pas spécifiquement temporelle, (ii) il n'est associé ni à une propriété systémique du temps verbal (comme c'est le cas du repérage anaphorique), ni à une propriété constante de ses emplois (comme c'est le cas de l'ancrage).

Le fait que ce processus ne soit pas intrinsèquement, mais seulement occasionnellement, associé aux temps verbaux pourrait éventuellement conduire à douter de son caractère anaphorique. Pourtant, rien dans la notion d'anaphore n'implique l'existence d'une propriété intrinsèque et constante de l'expression. Il existe certes de nombreuses expressions dont on peut dire qu'elles sont intrinsèquement anaphoriques ; mais l'anaphore n'en relève pas moins, fondamentalement, du domaine des emplois. Après tout, les syntagmes nominaux définis peuvent pratiquement tous donner lieu, selon le contexte dans lequel ils sont utilisés, à une interprétation anaphorique ou non anaphorique - ce dernier cas se présentant par exemple quand ils réfèrent sur le mode de la généricité (Corblin 1995).

Il est vrai qu'il est assez difficile de décrire la nature de l'information que reprend et proroge le processus de relais. Disons que les expressions qui marquent ce processus invitent à inférer un médiateur, différent de l'énonciateur, et à faire coïncider ce médiateur avec une instance de même statut et ayant déjà été précédemment mentionnée (en général au moyen d'une expression référentielle). Indirectement, et par le truchement du médiateur, c'est donc aussi une valeur référentielle que prorogent les expressions qui marquent ce processus de relais.

Ces trois processus anaphoriques pourraient être résumés comme suit :

- L'ancrage est le processus le plus général, car consubstantiel du fonctionnement des temps verbaux et de leur actualisation en discours. C'est sans doute la généralité de ce processus qui conduit certains linguistes à hésiter à le considérer comme anaphorique ${ }^{19}$.

- Le repérage anaphorique est spécifique à certains temps verbaux. Il en est un composant sémantique constant, " grammatical », et n'a donc en lui-même rien à voir avec la notion de contexte.

- Le relais, enfin, est un phénomène très général consistant dans le fait que certains temps verbaux peuvent, dans certaines circonstances, hériter localement d'une information médiative et la relayer dans la continuité du discours.

\footnotetext{
${ }^{19}$ Polémique qu'il n'est pas possible d'aborder ici.
} 


\subsection{Généralité du processus de relais}

Le relais médiatif est un fait discursif général qui ne concerne pas que les temps verbaux. Beaucoup d'autres expressions peuvent marquer ce type d'anaphore. Si l'on s'en tient aux verbes, il existe par exemple un phénomène bien connu en sémantique de l'espace, se produisant typiquement lorsqu'il s'agit de décrire le déplacement d'une « figure » relativement à un « site ». En régime narratif, la relation entre la « figure » et le «site » est souvent inversée, le « site » étant décrit comme mobile et la " figure » comme immobile (ce qui revient à traiter la " figure » comme un « site », et le « site » comme une «figure »). Cette inversion tient même du topos lorsqu'il s'agit de décrire une scène se passant dans un véhicule. Elle produit des formulations comme: des petites maisons roses défilaient dans le cadre de la fenêtre - l'église disparut derrière les rochers, etc. La fonction de cette inversion est évidemment médiative : elle reproduit les percepts d'un « sujet de conscience » en train de se déplacer - ce que Borillo (2012) appelle « perception inversée ».

Cette inversion produit généralement des énoncés empiriquement aberrants ou paradoxaux (comme des maisons qui se déplacent). Réciproquement, l'aberration sémantique fonctionne comme un indice de cette inversion. La lecture médiative, « subjective », a précisément pour effet de rétablir la normalité des situations décrites.

\section{BILAN ET CONSIDÉRATIONS SUR LE COUPLE ANAPHORE - DEIXIS}

Le principal objectif de cet article était d'analyser les différents mécanismes anaphoriques auxquels sont soumis les temps verbaux. Les exemples analysés ont permis de dégager trois processus relevant de l'anaphore: l'ancrage, le repérage anaphorique et le relais. Les deux premiers portent sur une information de localisation temporelle, le troisième sur la médiativité. Selon leur structure sémantique, certains temps verbaux ne sont soumis qu'à l'ancrage, d'autres à l'ancrage et au repérage anaphorique. Tous sont en revanche susceptibles, dans des circonstances adéquates, de relayer la médiativité ${ }^{20}$.

Concernant la question générale de l'anaphore et de la deixis, plus particulièrement de leur coexistence dans une même expression (anadeixis), les temps verbaux présentent aux moins deux particularités.

La première est que ces deux modes de repérage y sont logiquement ordonnés : le repérage déictique y est premier, il précède logiquement le repérage anaphorique. Une formulation procédurale et métaphorique de cette relation consisterait à dire que la « sortie » du repérage déictique fournit l' « entrée » du repérage anaphorique. C'est cette priorité de la deixis sur l'anaphore qui explique par exemple que le site temporel de l'ultériorité visée par le conditionnel (R) puisse être localisé dans le futur, quoique repéré depuis le passé $\left(\mathrm{r}_{1}\right)$.

La seconde particularité se rapporte à la conception même de la distinction entre anaphore et deixis. Certains auteurs considèrent, à la suite de Ehlich (1982) et Bosch (1983), que dans beaucoup d'expressions anadéictiques, les deux repérages « anaphore » et "deixis » ne relèvent pas de fonctionnements fondamentalement distincts, et ne sont que les deux extrêmes d'un continuum anadéictique. Ainsi Cornish (2011 et ici même) estime qu'il est possible de classer les anadéictiques sur une échelle allant du purement anaphorique au purement déictique. Or, une telle approche, si elle peut se justifier s'agissant des expressions nominales et de leurs proformes, est incompatible avec les observations faites dans le domaine des temps verbaux. La priorité de la deixis sur l'anaphore interdit à elle seule une telle conception. En d'autres termes, le fonctionnement de temps verbaux comme le plus-que-parfait ou le

${ }^{20} \mathrm{Y}$ compris les temps perfectifs, comme cela a été établi par plusieurs travaux (e.g. Ducrot 1980, Vetters 1994, Sthioul 2000, Bres 2003). 
conditionnel d'ultériorité ne saurait être caractérisé en ayant recours à un continuum anadéictique.

En résumé, les temps verbaux peuvent être le siège de quatre phénomènes phoriques : la deixis, l'anaphore par ancrage, l'anaphore par repérage, et l'anaphore par relais. Les deux premiers sont toujours présents dans tous les temps verbaux conjugables, le troisième est toujours présent mais dans certains temps verbaux seulement, et le quatrième est susceptible, dans certaines circonstances, de se manifester avec tous les temps verbaux.

\section{RÉFÉRENCES}

Apothéloz D. (2017). Reichenbach revisité. Verbum 39 (1), 5-30.

Apothéloz D. (2018). Une figure de l'oubli dans la narration: le passé simple dans les analepses. Romanica Cracoviensia 2, 49-60.

Apothéloz D. (2021). Les temps verbaux. In: Encyclopédie Grammaticale du Français. En ligne: encyclogram.fr

Arnauld A. \& Lancelot C. (1660). Grammaire générale et raisonnée. Paris : Chez Pierre Le Petit.

Ašić T., Bres J., Dodig M. \& Torterat F. (2017). Conditionnel temporel objectif et constructions non téléonomiques en français et en serbe. Travaux de linguistique 75 (2), 7-29.

Azzopardi S. (2011). Le futur et le conditionnel : valeur en langue et effets de sens en discours. Analyse contrastive espagnol / français. Université Paul Valéry, Montpellier III. Thèse de doctorat.

Bally Ch. (1912). Le style indirect libre en français moderne, II. Germanisch-Romanische Monatsschrift IV/10, 597-606.

Baranzini L. (2019). Le "récit de récit" à l'imparfait en italien : la piste évidentielle. Cahiers Chronos 30, 213-248.

Baranzini L., Ricci C. (2015) Semantic and pragmatic values of the Italian imperfetto: Towards a common interpretive procedure. Catalan Journal of Linguistics 14, 33-58.

Barceló G. J. \& Bres J. (2006). Les temps verbaux de l'indicatif en français. Paris : Ophrys.

Beauzée N. (1765). Article «Tems ». In : Encyclopédie ou Dictionnaire raisonné des sciences, des arts et des métiers, tome 16. Neufchastel : S. Faulche \& Cie, 96-117.

Béguelin M.-J. (ss la dir. de) (2000). De la phrase aux énoncés : grammaire scolaire et descriptions linguistiques. Bruxelles : De Boeck Duculot.

Berthonneau A.-M. \& Kleiber G. (1993). Pour une nouvelle approche de l'imparfait : l'imparfait, un temps anaphorique méronomique. Langages 112, 55-73.

Bertinetto P. M. (1986). Intrinsic and extrinsic temporal reference. On restricting the notion of 'reference time'. In: V. Lo Cascio \& C. Vet (eds), Temporal structure in sentence and discourse. Dordrecht: Foris, 41-78.

Borillo A. (2012). L'expression de déplacement fictif comme manifestation d'un discours narratif subjectif. In : L. de Saussure, A. Borillo, M. Vuillaume (éds), Grammaire, lexique, référence. Regards sur le sens. Berne : P. Lang, 45-58.

Bosch P. (1983). Agreement and anaphora, London, Academic Press.

Bres J. (2003). Temps verbal, aspect et point de vue : de la langue au discours. Cahiers de praxématique $41,55-84$.

Bres J. (2007). Sémantique de l'imparfait: dépasser l'aporie de la poule aspectuelle et de l'œuf anaphorique ? Éléments pour avancer. Cahiers Chronos 16, 23-46.

Bres J. (2012). Conditionnel et ultériorité dans le passé : de la subjectivité à l'objectivité. In : $3^{e}$ Congrès mondial de linguistique française, Lyon, 4-7 juillet 2012, 1719-1730. https://www.linguistiquefrancaise.org/cmlf-2012

Combettes B. (2008). Cohérence discursive et faits de langue : le cas du plus-que-parfait. Verbum 30 (23), 181-197.

Comrie B. (1985). Tense, Cambridge: Cambridge University Press.

Corblin F. (1995). Les formes de reprise dans le discours. Anaphores et chaînes de référence. Rennes: Presses Universitaires de Rennes.

Cornish F. (1987). Anaphoric pronouns: under linguistic control or signalling particular discourse representations? Journal of Semantics 5 (3), 233-260. 
Cornish F. (2011). 'Strict' anadeixis, discourse deixis and text structuring. Language Sciences 33 (5), 753-767.

Declerck R. (1986). From Reichenbach (1947) to Comrie (1985) and beyond. Towards a theory of tense. Lingua 70, 305-364.

De Mulder W. \& Vetters C. (1999). Temps verbaux, anaphores (pro)nominales et relations discursives. Travaux de linguistique 39, 37-58.

Desclés J.-P. \& Guentchéva Z. (2000). Énonciateur, locuteur, médiateur dans l'activité dialogique. In : A. Monod-Becquelin \& P. Erickson, Les rituels du dialogue. Paris : L’Harmattan, 79-112.

Ducrot O. (1980). Essai d'application : mais - les allusions à l'énonciation - délocutifs, performatifs, discours indirect. In: H. Parret et al., Le langage en contexte. Études philosophiques et linguistiques de pragmatique. Amsterdam : J. Benjamins, 487-576.

Ehlich K. (1982). Anaphora and deixis : same, similar, or different? In : R. J. Jarvella \& W. Klein (eds), Speech, place and action. New York : J. Wiley, 315-338.

Genette G. (1972). Figures III. Paris : Seuil.

Girard G. (dit Abbé Girard) (1747). Les vrais principes de la langue françoise, T. 2. Paris : Chez Le Breton.

Gosselin L. (1996). Sémantique de la temporalité en français. Louvain-la-Neuve, Duculot.

Gosselin L. (2005). Temporalité et modalité, Bruxelles : Duculot.

Gosselin L. (2017). Les temps verbaux du français : du système au modèle. Verbum 39 (1), 31-69.

Gosselin L. (2021). Aspect et formes verbales en français. Paris : Garnier.

Guentchéva Z. (1994). Manifestations de la catégorie du médiatif dans les temps du français. Langue française 102, 8-23.

Halliday M.A.K. \& Hasan R. (1976). Cohesion in English. London: Longman.

Hinrichs E. (1986). Temporal anaphora in discourses of English. Linguistics and Philosophy 9 (1), 63-82.

Keromnes Y. (1998). Aspect et anaphore. Cahiers Chronos 2, 1-19.

Kleiber G. (1993). Lorsque l'anaphore se lie aux temps grammaticaux. In : C. Vetters (éd.), Le temps, de la phrase au texte. Lille : Presses Universitaires de Lille, 117-166.

Kleiber G. (2003). Entre les deux mon cœur balance ou l'imparfait entre aspect et anaphore. Langue française 138, 8-19.

Korzen H. \& Nølke H. (2001). Le conditionnel : niveaux de modalisation. In: P. Dendale \& L. Tasmowski (éds), Le conditionnel en français. Metz : CELTED-Klincksieck, 125-146.

Koschmieder E. (1996/1929). Les rapports temporels fondamentaux et leur expression linguistique. Contribution à la question de l'aspect et du temps. Villeneuve-d'Ascq : Presses Universitaires du Septentrion. Trad. de: Zeitbezug und Sprache. Ein Beitrag zur Aspekt- und Tempusfrage. Wiesbaden: Springer, 1929.

Lhafi S. (2016). Rendements textuels du plus-que-parfait français et de son homologue espagnol. In : A. Berrendonner, M.-B. Mosegaard Hansen \& R. Zafiu (éds), Actes du $27^{e}$ Congrès international de linguistique et de philologie romane, Section 10, 133-144.

McCawley J. D. (1971). Tense and time reference in English. In: C. J. Fillmore \& D. T. Langendoen (eds), Studies in linguistic semantics. New York: Holt, Rinehart \& Winston, 96-113.

McCawley J. D. (1981). Note on the English perfect. Australian Journal of Linguistics 1, 81-90.

Molendijk A. (1996). Anaphore et imparfait: la référence globale à des situations présupposées ou impliquées. Cahiers Chronos 1, 109-123.

Nilsson-Ehle H. (1943). Le conditionnel "futur du passé" et la périphrase devait + infinitif. Studia neophilologica 16 (1), 50-88.

Partee B. H. (1973). Some structural analogies between tenses and pronouns in English. Journal of Philosophy 70, 601-609.

Partee B. H. (1984). Nominal and temporal anaphora. Linguistics and Philosophy 7, 243-286.

Portero Muñoz C. (2018). Tense switching in English narratives: an FDG perspective. Open Linguistics 4, 657-684.

Quine W. v. O. (1960). Word and object, Cambridge (Mass.): The M.I.T. Press.

Reichenbach H. (1947). Elements of symbolic logic. London: Macmillan.

Russell B. (1905). On denoting. Mind XIV (4), 479-493.

De Saussure L. (2003). Temps et pertinence. Bruxelles : De Boeck \& Larcier.

Sperber D. \& Wilson D. (1989). La pertinence. Paris : Minuit.

Sthioul B. (2000). Passé simple, imparfait et sujet de conscience. Cahiers Chronos 6, 79-93. 
Tasmowski-De Ryck L. (1985). L’imparfait avec et sans rupture. Langue française 67, 59-77.

Tesnière L. (1969). Éléments de syntaxe structurale, Paris, Klincksieck.

Vet C. (1996a), «Anaphore et deixis dans le domaine temporel », Cahiers Chronos 1, 147-163.

Vet C. (1996b). Aspect, anaphore et interprétation du discours. HERMES - Journal of Language and Communication in Business 16, 93-106.

Vet C. (1999). Les temps verbaux comme expressions anaphoriques. Travaux de linguistique 39, 113130.

Vetters C. (1993). Temps et deixis. In : C. Vetters (éd.), Le temps, de la phrase au texte. Lille : Presses Universitaires de Lille, 85-115.

Vetters C. (1994), "Free indirect speech in French", in C. Vet \& C. Vetters (eds), Tense and aspect in discourse, Berlin, Mouton de Gruyter, 179-225.

Vetters C. (1996). Temps, aspect et narration. Amsterdam : Rodopi.

Vetters C. (1998). Les “temps" du verbe. Réflexions sur leur temporalité et comparaison avec la référence (pro)nominale. In : Sv. Vogeleer, A. Borillo, C. Vet \& M. Vuillaume (éds), Temps et discours. Louvain-La-Neuve : Peeters, 11-43.

Wilhelm R. (2009). Der conditionnel narratif. Ein neues Erzähltempus und sein Gebrauch in der französischen Literatursprache. Romanische Forschungen 121, 133-161.

Wilmet M. (1997). Grammaire critique du français. Bruxelles : De Boeck-Duculot.

Yvon H. (1951). Convient-il de distinguer dans le verbe français des temps relatifs et des temps absolus ? Le français moderne 19, 265-276. 